crease nosocomial infections in an intermediate care unit. Crit Care Med 2004;32(2):358-363.

9. Levchenko AI, Boscart VM, Fernie GR. The effect of automated monitoring and real-time prompting on nurses' hand hygiene performance. Comput Inform Nurs 2013;31(10):498-504.

\section{Anatomic Sites of Colonization with Community-Associated Methicillin- Resistant Staphylococcus aureus}

Methicillin-resistant Staphylococcus aureus (MRSA) has emerged over the past 20 years as a cause of infections in community populations, so-called community-associated MRSA (CA-MRSA). By pulsed-field gel electrophoresis (PFGE) subtyping, USA300 is the most common CA-MRSA strain in the United States. It has been suggested that the colonization dynamics for CA-MRSA may be different than those for traditional MRSA strains, ${ }^{1}$ with extranasal colonization potentially playing a role in CA-MRSA transmission and infection. ${ }^{1}$

Another distinguishing characteristic of USA300 MRSA strains is greater susceptibility to non- $\beta$-lactam antibiotics compared with healthcare-associated MRSA strains. However, multidrug-resistant (MDR) USA300 strains have been described, largely among patients infected with human immunodeficiency virus (HIV) and men who have sex with men (MSM). ${ }^{2}$ Also of concern, resistance to common decolonizing agents, such as mupirocin ${ }^{2}$ and chlorhexidine gluconate $(\mathrm{CHG}),{ }^{3}$ has been reported in CA-MRSA.

The objectives of this study were to examine the phenotype of USA300 MRSA strains, the prevalence of the qacA/B gene in this population, and the anatomic sites of colonization by PFGE pattern.

We previously reported on the prevalence of nasal and extranasal CA-MRSA colonization among inpatients (374 HIV infected and 371 HIV negative) at Stroger Hospital of Cook County $(\mathrm{CCH})$, the major safety net hospital in Chicago. ${ }^{4}$ As described elsewhere, nasal and extranasal (throat, axilla, inguinal, perirectal, and chronic wound, if present) surveillance swab specimens were collected from patients within 72 hours of admission from March 2011 to April 2012; cultures were processed with broth enrichment. ${ }^{4}$ Sex was recorded, and enrolled men were asked whether they identified themselves as MSM. Genotypic analysis with PFGE was performed on all identified MRSA isolates. Results were interpreted as described by McDougal et al. ${ }^{5}$

Confirmed MRSA isolates had antibiotic susceptibility determined (MicroScan Walkaway System, Siemens Healthcare Diagnostics). For USA300 MRSA strains, MDR was defined as resistance to 4 or more non- $\beta$-lactam antibiotic classes. High-level mupirocin resistance was assessed using disk diffusion. ${ }^{6}$

Carriage of qacA and qacB genes, which code for efflux pumps associated with increased minimum inhibitory concentrations of $\mathrm{CHG},{ }^{7}$ was assessed using real-time polymerase chain reaction, as described previously. ${ }^{8}$

A $\chi^{2}$ test was used to examine the association of PFGE patterns and colonization sites, with Fisher exact test used for small samples. SAS software (ver. 9.2; SAS Institute) was used for statistical analysis. The study was approved by the institutional review board of $\mathrm{CCH}$ and Rush University Medical Center.

We observed that following the nares, the perirectal area was the second most common site of colonization (58\% of colonized individuals). Prevalence of extranasal and exclusive extranasal colonization was not significantly different between patients colonized with USA300 or non-USA300 strains (Table 1). However, the average number of sites colonized was significantly higher for USA300 versus non-USA300 strains (2.8 [standard deviation (SD), 1.51] and 2.2 [SD, 1.48], respectively; $P=.049$ ). Inguinal, perirectal, and concomitant inguinal and perirectal colonization were all significantly associated with colonization with the USA300 strain type in comparison to non-USA300 MRSA strains (Table 1). Inguinal or perirectal MRSA colonization was found more often in men $(63 / 480 ; 13 \%)$-MSM (odds ratio [OR], 2.2 [95\% confidence interval (CI), 1.1-4.2]; $P=.02$ ) and heterosexual men (OR, 1.8 [95\% CI, 1.02-3.2]; $P=.04)$-than in women (20/265; 8\%; OR, 1.9 [95\% CI, 1.1-3.1]; $P=.02$ ).

There were 5 individuals who had an MRSA infection at the time of enrollment, and they were all found to have colonization with MRSA. Four of these individuals had skin and soft tissue infections and were colonized with the USA300 strain type, and 1 individual had a bloodstream infection and was colonized with a non-USA300 strain type. Excluding chronic wound cultures, each of these individuals had 3-5 sites of MRSA colonization, suggesting a significant level of extranasal colonization and colonization burden for individuals infected with MRSA.

Of the colonized individuals, $3.4 \%$ carried high-level mupirocin-resistant strains (1 USA100, 2 USA500, 1 USA300). Of the individuals colonized with USA300 MRSA strains, $4(5 \%)$ carried MDR strains. There were 117 MRSA isolates evaluated for the presence of the qac $\mathrm{A} / \mathrm{B}$ genes; all were negative.

We examined colonization and molecular characteristics of CA-MRSA isolates collected from patients seeking care at the major safety net hospital in Chicago. We found that inguinal and perirectal colonization was more common with the USA300 strain type than with non-USA300 MRSA strains. In addition, highly antibiotic-resistant USA300 MRSA strains were rare, and none of the MRSA isolates collected over a 14-month study period were found to harbor the qac $\mathrm{A} / \mathrm{B}$ genes.

We observed that males-both heterosexual males and MSM-had a higher prevalence of inguinal and perirectal MRSA colonization in comparison to females. Similarities observed in colonization patterns between MSM and heterosexual males suggest that perhaps social, hormonal, skin 
TABLE 1. Association of Pattern of Anatomic Site of Colonization and Pulsed-Field Gel Electrophoresis Profile among Individuals Colonized with Community-Associated Methicillin-Resistant Staphylococcus aureus (CA-MRSA)

\begin{tabular}{|c|c|c|c|c|}
\hline Anatomic site of colonization & USA300 $(n=79)^{\mathrm{a}}$ & Non-USA300 $(n=36)$ & OR $(95 \% \mathrm{CI})$ & $P$ \\
\hline Anterior nares & $50(63)$ & $21(58)$ & $\ldots$ & .61 \\
\hline Throat & $35(44)$ & $16(44)$ & $\ldots$ & .99 \\
\hline Axilla & $31(39)$ & $12(33)$ & $\ldots$ & .54 \\
\hline Inguinal & $49(62)$ & $15(42)$ & $2.3(1.02-5.11)$ & .04 \\
\hline Perirectal & $52(66)$ & $15(42)$ & $2.7(1.2-6.06)$ & .015 \\
\hline Inguinal and perirectal region & $40(51)$ & $9(25)$ & $3.1(1.28-7.37)$ & .01 \\
\hline Wound & $6(8)$ & $1(3)$ & $\ldots$ & .43 \\
\hline Extranasal colonization & $74(94)$ & $32(89)$ & $\ldots$ & .46 \\
\hline Exclusive extranasal colonization & $29(37)$ & $15(42)$ & $\ldots$ & .61 \\
\hline Sites colonized, average (SD) & $2.8(1.51)$ & $2.2(1.48)$ & $\ldots$ & .049 \\
\hline
\end{tabular}

note. Data are no. (\%) of patients, unless otherwise indicated. Any extranasal colonization was defined as the presence of CA-MRSA extranasal colonization irrespective of anterior nares culture results. Exclusive extranasal colonization was defined as CA-MRSA colonization at extranasal sites and negative anterior nares cultures for CA-MRSA. CI, confidence interval; OR, odds ratio; SD, standard deviation.

a Two individuals were colonized with both USA300 and non-USA300 strains and were excluded from the comparison of USA300 to non-USA300 strains. Therefore, the total number of patients used in the analysis was 115 .

biology, or genetic differences between sexes play a role in colonization dynamics rather than sexual orientation. ${ }^{9}$

The absence of $q a c \mathrm{~A} / \mathrm{B}$ genes among MRSA isolates in the population we studied is consistent with other reports in the United States. ${ }^{10}$ In contrast to reports in San Francisco, ${ }^{2}$ MDR USA300 strains were relatively rare in our population, which comprised a diverse group of HIV-infected and HIV-negative individuals. However, continued surveillance of antibiotic resistance patterns is needed to understand the evolving epidemiology of USA300 strains as well as to inform empiric therapeutic decisions.

Our study is limited in that we did not assess frequency of mupirocin or $\mathrm{CHG}$ use in our population, although $\mathrm{CCH}$ does not have mupirocin on its antibiotic formulary and CHG bathing use has been limited to intensive care unit and preoperative patients. In addition, we relied on self-report of MSM status, which could lead to recall bias. Finally, we performed PFGE on 1 MRSA colony morphotype per body site and therefore may have failed to detect coexistent minority subpopulations.

In summary, our study highlights that inguinal and perirectal colonization appears to be more frequent with the USA300 strain type and that sex may play a role in location of extranasal CA-MRSA colonization. Patients with clinical MRSA infections appeared to be those with more sites of MRSA colonization. Although mupirocin resistance and presence of $q a c \mathrm{~A} / \mathrm{B}$ were uncommon, continued monitoring of MRSA prevalence and resistance is warranted.

\section{ACKNOWLEDGMENTS}

We thank Karen Lolans (Rush University Medical Center), Amanda Grasso (Stroger Hospital of Cook County), Andrej Spec (Rush University Medical Center), Janki Patel (Rush University Medical Center), Lisa Kurien (Rush
University Medical Center), and Rosie Lyles-Banks (Stroger Hospital of Cook County) for their help with this project.

Financial support. This project was supported by grant K23AI085029 (principal investigator: K.J.P.) from the National Institute of Allergy and Infectious Diseases. The content is solely the responsibility of the authors and does not necessarily represent the official views of the National Institute of Allergy and Infectious Diseases or the National Institutes of Health. This project was also supported by the Centers for Disease Control and Prevention Epicenter Grant Cooperative Agreement 1U54CK000161 (principal investigator: R.A.W.).

Potential conflicts of interest. All authors report no conflicts of interest relevant to this article. All authors submitted the ICMJE Form for Disclosure of Potential Conflicts of Interest, and the conflicts that the editors consider relevant to this article are disclosed here.

Kyle J. Popovich, MD, MS; ${ }^{1,2}$ Alla Aroutcheva, MD, PhD; ${ }^{1,2}$ Bala Hota, MD, MPH; ${ }^{1,2}$ Kathleen G. Beavis, MD; Mary K. Hayden, MD; ${ }^{1}$ Robert A. Weinstein, MD $^{1,2}$

Affiliations: 1. Rush University Medical Center, Chicago, Illinois; 2. Stroger Hospital of Cook County, Chicago, Illinois; 3. University of Chicago Medicine, Chicago, Illinois.

Address correspondence to Kyle J. Popovich, MD, MS, 600 South Paulina, Suite 143, Chicago, IL 60612 (kyle_popovich@rush.edu).

Received February 12, 2014; accepted April 2, 2014; electronically published July 25, 2014.

Infect Control Hosp Epidemiol 2014;35(9):1192-1194

(C) 2014 by The Society for Healthcare Epidemiology of America. All rights reserved. 0899-823X/2014/3509-0017\$15.00. DOI: 10.1086/677627

\section{REFERENCES}

1. Miller LG, Diep BA. Clinical practice: colonization, fomites, and virulence: rethinking the pathogenesis of community-associated methicillin-resistant Staphylococcus aureus infection. Clin Infect Dis 2008;46(5):752-760. 
2. Diep BA, Chambers HF, Graber CJ, et al. Emergence of multidrug-resistant, community-associated, methicillin-resistant Staphylococcus aureus clone USA300 in men who have sex with men. Ann Intern Med 2008;148(4):249-257.

3. Fritz SA, Hogan PG, Camins BC, et al. Mupirocin and chlorhexidine resistance in Staphylococcus aureus in patients with community-onset skin and soft tissue infections. Antimicrob Agents Chemother 2013;57(1):559-568.

4. Popovich KJ, Hota B, Aroutcheva A, et al. Community-associated methicillin-resistant Staphylococcus aureus colonization burden in HIV-infected patients. Clin Infect Dis 2013;56(8): 1067-1074.

5. McDougal LK, Steward CD, Killgore GE, Chaitram JM, McAllister SK, Tenover FC. Pulsed-field gel electrophoresis typing of oxacillin-resistant Staphylococcus aureus isolates from the United States: establishing a national database. J Clin Microbiol 2003;41(11):5113-5120.

6. Clinical and Laboratory Standards Institute (CLSI). Performance Standards for Antimicrobial Susceptibility Testing: 21st Informa- tional Supplement. Wayne, PA: CLSI, 2011. CLSI document M100-S21.

7. Horner C, Mawer D, Wilcox M. Reduced susceptibility to chlorhexidine in staphylococci: is it increasing and does it matter? $J$ Antimicrob Chemother 2012;67(11):2547-2559.

8. Hayden MK, Lolans K, Li H, et al. Chlorhexidine gluconate (CHG) susceptibility of methicillin-resistant Staphylococcus aureus (MRSA) isolates from a multi-state study of adult intensive care unit (ICU) patients. In: 20th Annual Scientific Meeting of the Society for Healthcare Epidemiology of America. April 1-4, 2011; Dallas, TX. Abstract 369.

9. Zanger P, Nurjadi D, Gaile M, Gabrysch S, Kremsner PG. Hormonal contraceptive use and persistent Staphylococcus aureus nasal carriage. Clin Infect Dis 2012;55(12):1625-1632.

10. McGann P, Kwak YI, Summers A, Cummings JF, Waterman PE, Lesho EP. Detection of qacA/B in clinical isolates of methicillinresistant Staphylococcus aureus from a regional healthcare network in the eastern United States. Infect Control Hosp Epidemiol 2011;32(11):1116-1119. 